\title{
Research of Shielding Effectiveness of an Elastic Shield Made of Conductive Fabric to Ensure HEMP Protection of Electronic Equipment
}

\author{
Vladimir Gurevich, Ph.D. \\ Central Electrical Laboratory Israel Electric Corp., Haifa, Israel
}

*Corresponding Author: Vladimir Gurevich, Central Electrical Laboratory Israel Electric Corp., Haifa, Israel

\begin{abstract}
The article discusses the research findings regarding shielding capacity of two types of conductive fabrics within $100 \mathrm{kHz}-100 \mathrm{MHz}$ range, which corresponds to HEMP. SEMS devices, equipped with various types of antennas, (as part of the device) were used to measure the efficiency of shielding capacity. The article also describes a possibility of using a circular loop antenna to measure the attenuation of an electric field, and not for measuring a magnetic field only, as stated by the SEMS device manufacturer.
\end{abstract}

Keywords: shielding; conductive fabric; loop antennas; electric field measurement; HEMP; EMP

\section{INTRODUCTION}

The issue of protection of a power system's electronic equipment (digital devices of relay protection and automation, remote control and communication systems, auxiliary power DC systems, etc.) from the electromagnetic pulse of a high-altitude nuclear explosion (HEMP) is very relevant. Various solutions of this problem, suitable for wide practical use, are covered in the author's monograph [1]. None of the available individual protection means ensures the adequate level of protection from EMP. The adequate level of protection can be achieved by the aggregate of these means only.

One of the options is represented by conductive fabric, which is used to shield the open bottom part of control cabinets with electronic equipment at the entry point of multiple control cables. It can also be used for shielding doors, windows, lining of internal surfaces of premises being shielded, as well as cabinets and tents at storages of sensitive electronic equipment, etc.

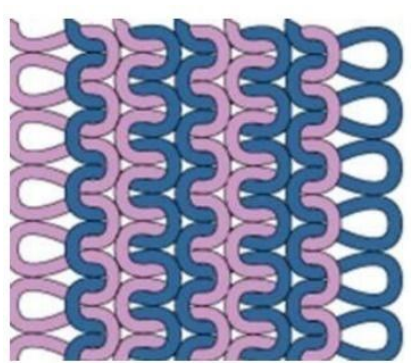

Knitted Fabric

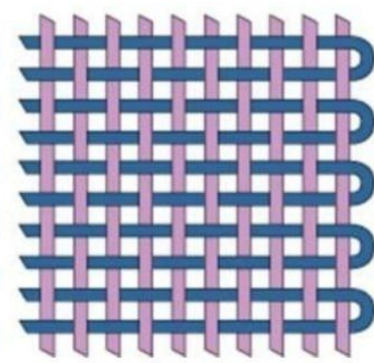

Woven Fabric

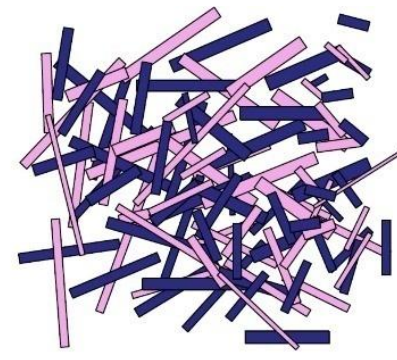

Non-Woven

Fabric

Figure1. Varieties of fabrics

These fabrics are produced by different companies, such as Holland Shielding Systems, Metal Textiles Corp., V Technical Textiles Inc., Swift Textile Metalizing, Seiren Electronics Materials, Saint year Electronic, Aaronia AG, P\&P Technology and others.

There are multiple varieties of fabrics produced under alternative technologies, Fig. 1. Woven fabric and non-woven materials are used in conductive fabric production. Textile materials can also be nonwoven if they are produced without weaving, i.e. their individual fibers are connected by a type of adhesive. Nevertheless, fibers used for creation of woven and non-woven textiles are obtained by spattering of a micron layer of conductive metals, such as copper, silver and nickel on a thin polymer International Journal of Research Studies in Electrical and Electronics Engineering (IJRSEEE) Page | 1 
fiber (e.g. made of polyamide resin or polyester). These textiles resemble dense fabric, but with traces of metal.
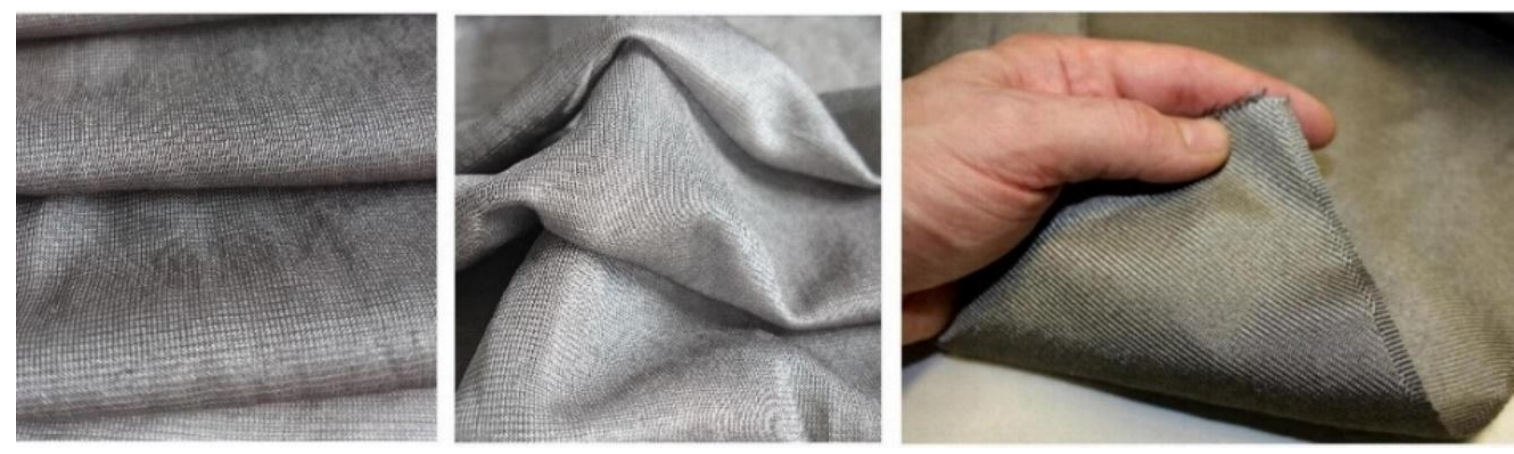

Figure2. Conductive fabric

There are also polymer films with metal coating, which are usually produced in the form of bands. They are thinner compared to textile materials, but the latter are also very thin $(0.1-0.25 \mathrm{~mm})$. It should be noted that the thickness of fabric has nothing to do with that of the conductive layer. The thinness of conductive spatter determines the frequency range in which these materials demonstrate efficient absorption of electromagnetic emission, since the shielding capacity of the material is directly related to the depth of penetration $(\Delta)$ of the electromagnetic wave into the material:

$$
\Delta=503 \cdot \sqrt{\frac{\rho}{\mu \cdot f}}
$$

\section{Where: $\Delta$ - the skin depth,}

$\boldsymbol{\rho}$ - specific electrical resistance of metal,

$\boldsymbol{\mu}$ - magnetic permeability of metal,

$f$ - radiation frequency.

Table1. Skin depth of copper and aluminum.

\begin{tabular}{|c|c|c|}
\hline \multirow{2}{*}{ Frequency, MHz } & \multicolumn{2}{|c|}{ Skin depth, mm } \\
\cline { 2 - 3 } & Copper & Aluminum \\
\hline 0.001 & 2.09 & 2.6 \\
\hline 0.01 & 0.66 & 0.83 \\
\hline 0.1 & 0.21 & 0.26 \\
\hline 1.0 & 0.066 & 0.083 \\
\hline 10 & 0.02 & 0.026 \\
\hline 100 & 0.0066 & 0.0083 \\
\hline 1000 & 0.0021 & 0.0026 \\
\hline
\end{tabular}

The skin depth is the thickness of the surface layer of metal, where the density of the electromagnetic field is reduced in $\mathrm{e}=2.718$ times. According to [2], this layer will account for almost $86 \%$ of energy coming from the surface. Table 1 shows the data on the skin depth of copper and aluminum.

Obviously, the operating range of protecting a shield covered with a very thin conductive layer falls within hundreds and thousands of megahertz. Indeed, various types of conductive fabric and films are produced for this frequency range. However, $96 \%$ of the E1 component of HEMP falls within the 100 $\mathrm{kHz}-100 \mathrm{MHz}$ [3] range, which is beyond the designed frequency range of conductive fabrics. When asking a direct question whether or not this fabric can be used in HEMP's frequency range, the manufacturers failed to provide any precise and self-explanatory answer.

The situation becomes even worse as the shielding efficiency is determined by not only absorption of electromagnetic emission by the shielding material, but also by reflection of this emission from the material's surface, which is stipulated by conductivity of its surface layer and has nothing to do with thickness.

Lack of reliable information raised a question whether or not conductive fabric can be used in the HEMP's frequency range, which is significantly different from what is declared by manufacturers.

International Journal of Research Studies in Electrical and Electronics Engineering (IJRSEEE) Page $\mid 2$ 


\section{Measuring Of Shielding EfFectiveness Of Conductive Fabrics}

For research purposes, two types of fabrics were picked up. Samples of these fabrics were supplied to the author by two different manufacturers.

Since the idea was not to test the material, but the shield made of it, the purpose of the research equipment was to measure the shielding capacity of enclosures (cabinets, chambers, walls and rooms). Measurements were performed by SEMS devices (Fig. 3) manufactured by MPB Electronic (Italy), with a set of various antennas (Fig. 4) suitable for different frequency ranges.

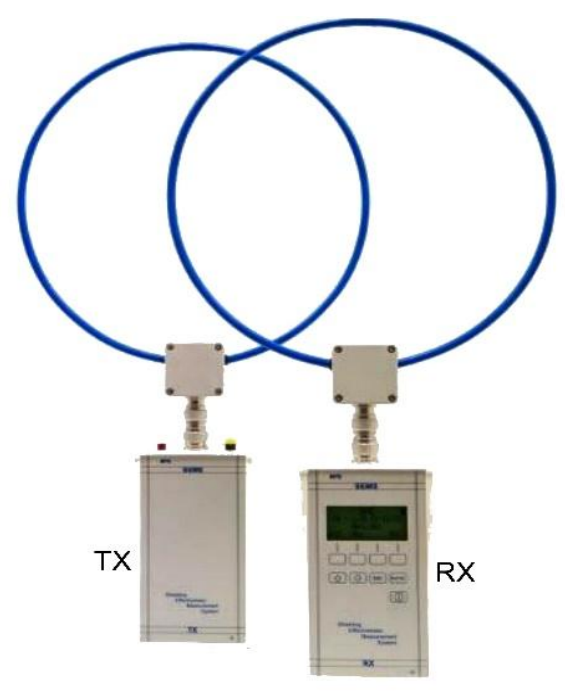

Figure3. SEMS device to measure shielding efficiency. TX - transmitter, $R X$ - receiver

The device includes a programmable high-frequency transmitter and receiver (Fig. 3) mounted on special tripods and equipped with a remote system of synchronization and data exchange. One of these devices should be placed inside the enclosure being tested, whereas the other is placed outside. The shielding capacity of the enclosure is determined by the intensity difference between the emitted and the received signal.

The problem is that devices available in the market are designed for frequencies ranging from hundreds to thousands of megahertz. Manufacturer's specifications $(0,1-300 \mathrm{MHz})$ of selected SEMS devices were expected to fully satisfy our requirements to the frequency range. However, the frequency ranges of antennas employed in this device (Fig. 4) fell very much beyond the required specifications.
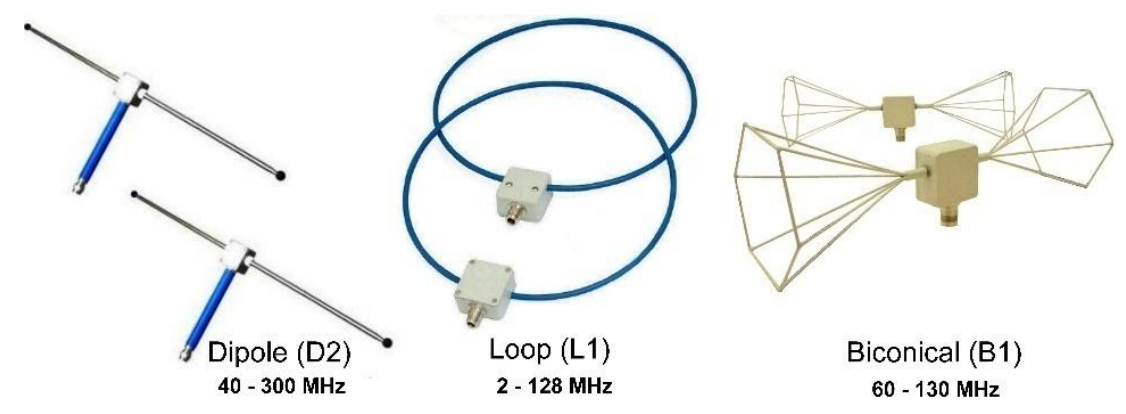

Figure4. Antennas of SEMS device

It was only the circular loop antenna (L1 type) that demonstrated the operating range close to what was required. Nevertheless, according to the manufacturer, this antenna is designed to measure the attenuation of a magnetic field, but not an electric field. Since non-ferromagnetic materials (copper, aluminum, silver) do not shield a magnetic field, (conductive fabric is a non-ferromagnetic material, which is transparent for a magnetic field) this seemingly suitable (in terms of the frequency range) loop antenna cannot be used for testing the shield made of conductive fabric. This restriction for a circular loop antenna use seemed to us unjustified, yet the SEMS manufacturer did not provide any straightforward answer whether this antenna can be used to measure the shielding efficiency of a 
magnetic field, rather than an electric field. Thus, a series of tests were performed with different antennas to measure the shielding efficiency of the protective enclosure (Fig. 5) made of Shieldex Nora Dell conductive fabric within the closest $(100 \mathrm{kHz}-100 \mathrm{MHz})$ frequency range (Fig. 6). This woven material manufactured by StatexProduktions\& Vertriebs GmbH (Germany) is made of nylon fibers coated with nickel, copper and silver.

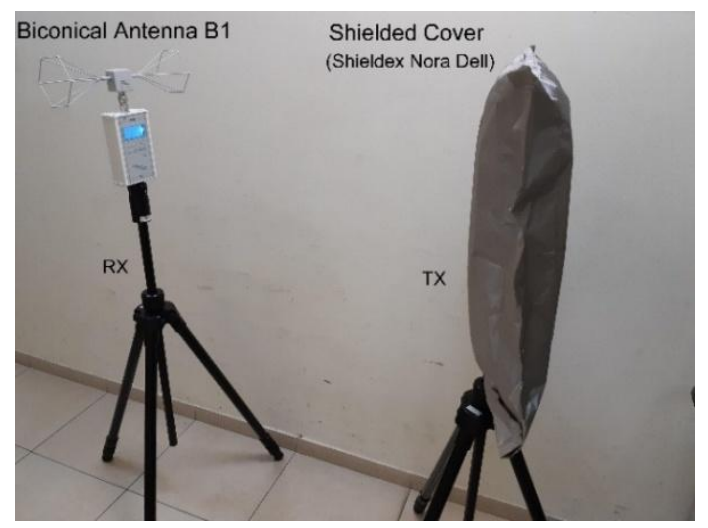

Figure5. Measuring the shielding capacity of a case made of conductive fabric with the SEMS device.

Moreover, since the manufacturer designed these antennas for an alternative frequency range, each set of antennas (both on the receiver and on the transmitter) was initially tested in the air to measure the real frequency range in which this set ensures zero attenuation during measurements, i.e. the range which can be used for shielding efficiency research in the future. This range is shown in Fig. 6.

The test results of a biconical antenna (Fig. 6A) show clear irregularity of its specifications within a narrow range $(30-40 \mathrm{MHz})$, which can be explained by the specific design of the SEMS device. This irregularity is repeated and gets stronger later upon measuring the shielding efficiency by the conductive fabric. Thus, this narrow area of the frequency range can be neglected as it has nothing to do with the shielding capacity of the material under question.

The specified frequency range $(140-180 \mathrm{MHz})$ of a dipole aerial with three elements falls beyond the range we are interested in and thus it is of no concern.
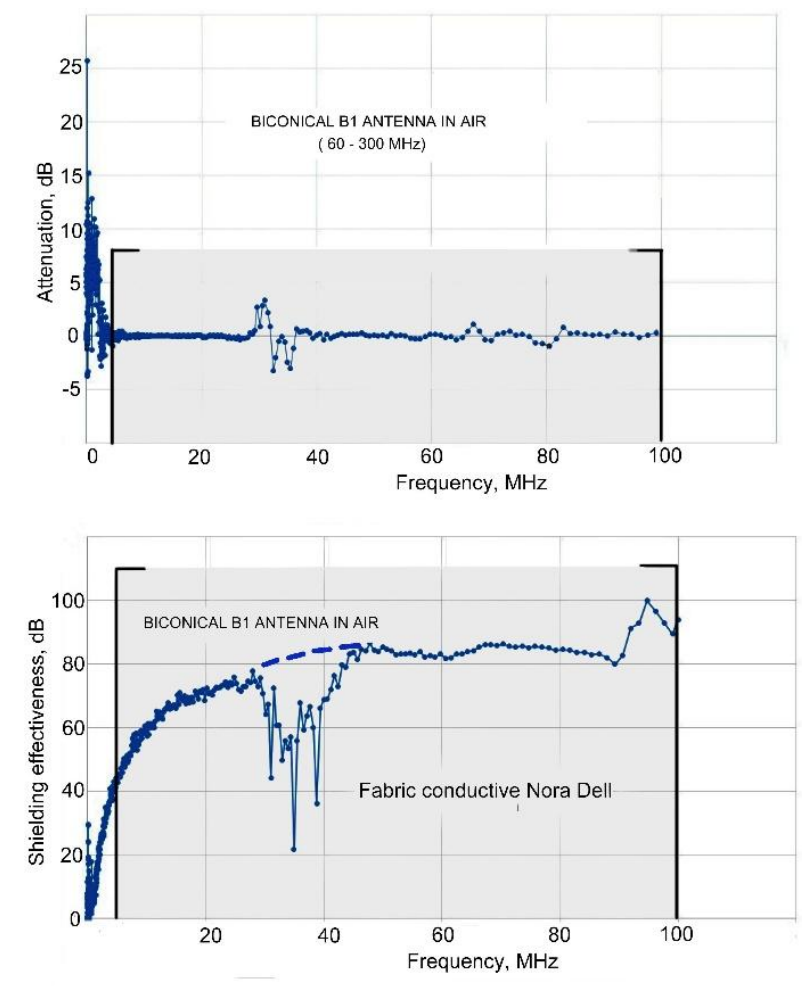

Figure6a. Determination of operating frequency range of a biconical B1 antenna in the air (above) and shielding efficiency provided by Shieldex Nora Dell fabric measured with this antenna. 

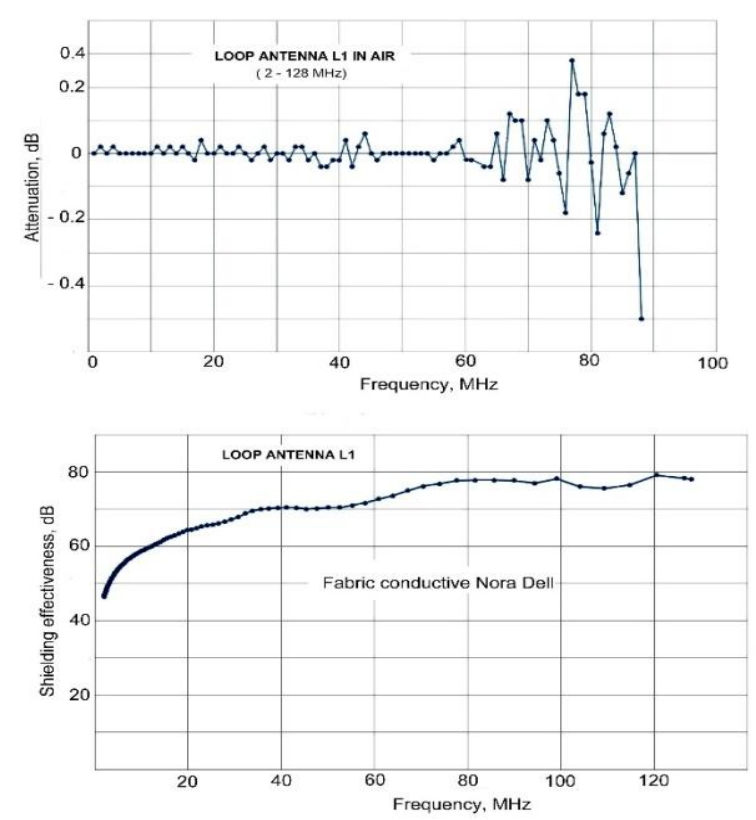

Figure6b. Determination of operating frequency range of a circular loop antenna L1 in the air (above) and shielding efficiency provided by Shieldex Nora Dell fabric measured with this antenna.

Notwithstanding the restrictions introduced in the manufacturer's technical manuals stipulating that this antenna can only be used for measuring the magnetic field shielding efficiency, it proved to be comparably good for measuring the electric field shielding efficiency (otherwise, the shielding efficiency of non-ferromagnetic conductive fabric measured with this antenna should have been equal to zero). Moreover, while measuring the shielding efficiency of the same material by these two types of antennas, the obtained values appeared to be very close (Fig. 7). Using standard conversions offered by MS Excel, the test curves obtained by two antennas were fit as corresponding functions (shown in Fig. 7). This nonlinear logarithmic regression can be described by coefficient of determination $\mathrm{R}^{2}$, (where $\mathrm{R}$ is the correlation coefficient) which shows the degree of test data approximation using a certain theoretic model. In this case, the value of $\mathrm{R}^{2}$ is very high. This means that the regression model is rather accurate in describing the experimental data.

Figure 7 shows that for the frequency range under investigation, the curves obtained with this model for circular loop (L1) and biconical (B1) antennas are situated close to each other. This allows to conclude that circular loop antennas can freely be used for measuring electric field shielding efficiency within HEMP's frequency range with rather acceptable accuracy.

Upon establishing that a circular loop antenna can be used for measuring the shielding efficiency of an electric field, direct measurements of shielding efficiency provided by another type of conductive fabric - SC.NICU (with polymer fiber coated with nickel and copper, without silver), manufactured by Soliani EMC, Italy (Fig.8) - were performed.

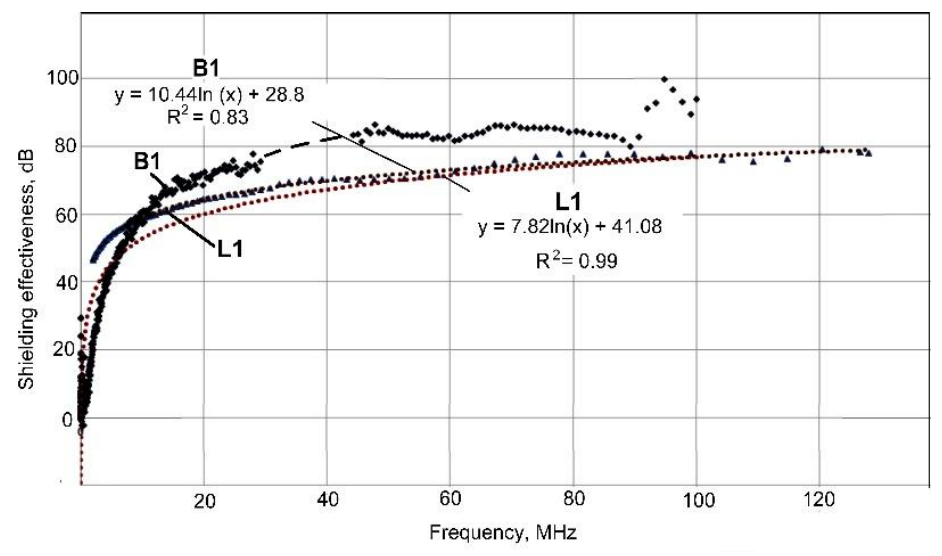

Figure7. Regression models built upon measuring the shielding efficiency of an electric field using a circular loop antenna LI and a biconical antenna Bl.

International Journal of Research Studies in Electrical and Electronics Engineering (IJRSEEE) Page 5 


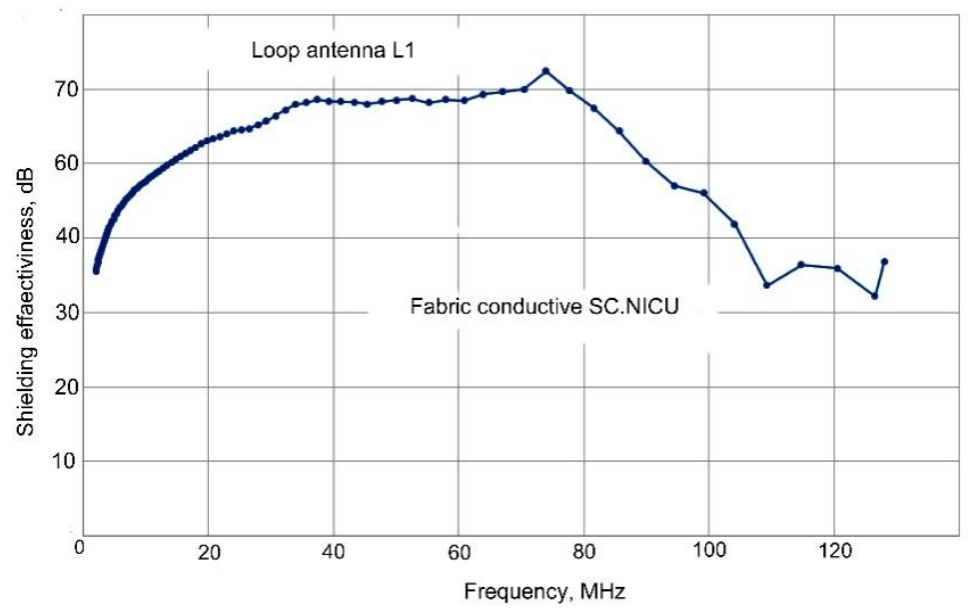

Figure8. The shielding efficiency of an electric field by SI.NICU conductive fabric, measured by means of a circular loop antenna L1.

Upon confirming that a circular loop antenna can be used for measuring the shielding efficiency of an electric field, another research of two types of conductive fabrics in the low frequency range was performed using the low-frequency model of a circular loop antenna (L2) that is one of the additional antennas from the SEMS antennas kit (Fig. 9).
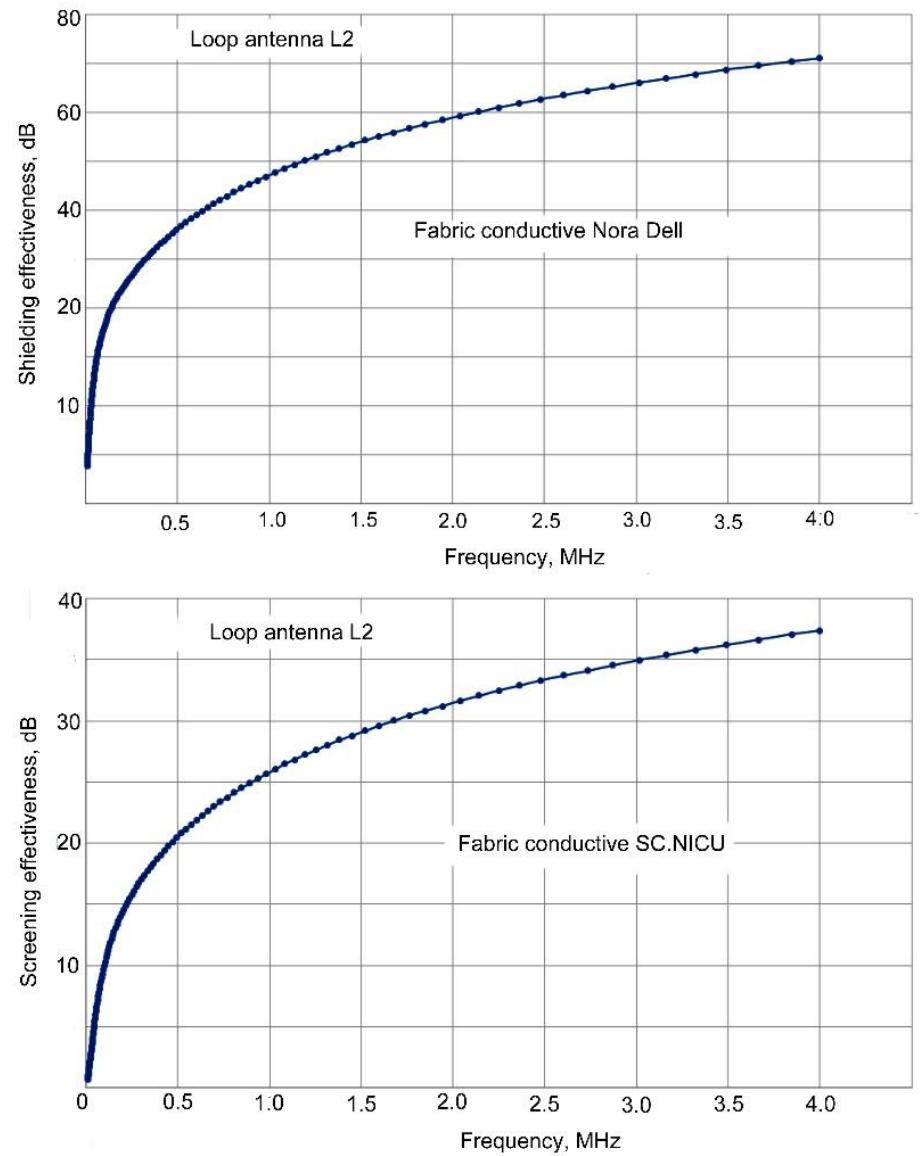

Figure9. Shielding efficiency in the low-frequency part of the range by two conductive fabrics: Shieldex Nora Dell and SC.NICU.

\section{CONCLUSION}

The findings suggest that conductive woven materials are rather efficient shields suitable for HEMP protection of electronic equipment. Among two materials being tested, the fabric containing silver as one of the fiber's coating layer (Shieldex Nora Dell) performed better (especially in the low-frequency part of the range) compared to the fabric without silver coating. It is noteworthy that silver-containing International Journal of Research Studies in Electrical and Electronics Engineering (IJRSEEE) Page 6 
material is significantly more expensive ( USD 77 per linear meter, $130 \mathrm{~cm}$ wide) than the material that does not contain silver ( USD 46 per the same linear meter). In a case where conductive fabric is used in combination with other protection means (as a rule) and not as a single protection element, some reduction of shielding capacity with significant cost reduction of the material is fairly acceptable.

\section{ACKNOWLEDGMENT}

The author would like to express his appreciation to Eng. Alexander Mailis is for his help with measurement tests.

\section{REFERENCES}

[1] Gurevich V.I. Electromagnetic pulse of high-altitude nuclear explosion and protection against it. - M.: Infra-Ingeneria, 2018. pp. 516.

[2] Industrial electro heat installations / N. M. Nekrasova, L.S. Katsevich, I. P. Yevtyukova. - M.: Gosenergoizdat. 1961, pp. 415.

[3] IEC 61000-2-9 Electromagnetic compatibility (EMC). Part 2: Environment - Section 9: Description of HEMP environment - Radiated disturbance, 1996

\section{AUTHOR'S BIOGRAPHY}

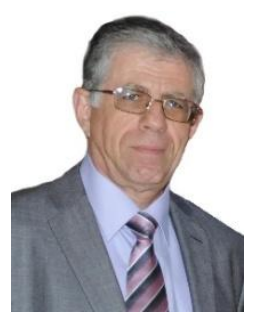

Vladimir I. Gurevich, was born in Kharkov, Ukraine, in 1956. He received an M.S.E.E. degree (1978) at the Kharkov Technical University, named after P. Vasilenko, and a Ph.D. degree (1986) at Kharkov National Polytechnic University. His employment experience includes: teacher, assistant professor and associate professor at Kharkov Technical University, and chief engineer and director of Inventor, Ltd. In 1994, he arrived in Israel and works today at Israel Electric Corp. as a senior specialist and Head of section of the Central Electric Laboratory.

He is the author of more than 200 professional papers and 15 books and holder of nearly 120 patents in the field of electrical engineering and power electronics. In 2006 he was Honorable Professor with the Kharkov Technical University.

Citation: Vladimir Gurevich, "Research of Shielding Effectiveness of an Elastic Shield Made of Conductive Fabric to Ensure HEMP Protection of Electronic Equipment", International Journal of Research Studies in Electrical and Electronics Engineering, 5(1), pp 1-7. DOI: http://dx.doi. org/10.20431/2454-9436.0501001

Copyright: (C) 2019 Authors. This is an open-access article distributed under the terms of the Creative Commons Attribution License, which permits unrestricted use, distribution, and reproduction in any medium, provided the original author and source are credited. 